\title{
HABITAT PREFERENCES, DISTRIBUTION AND ANATOMY OF THE CLASPING-LEAVED PONDWEEDS OF TURKEY
}

\author{
Necati BAYINDIR, Nursel İKİNCİ* \\ Bolu Abant İzzet Baysal University, Department of Biology, 14280 Bolu, TURKEY
}

Cite this article as:

Bayındır N. \& İkinci N. 2020. Habitat Preferences, Distribution and Anatomy of the Clasping-leaved Pondweeds of Turkey. Trakya Univ J Nat Sci, 21(2): 95-106, DOI: $10.23902 /$ trkjnat.746096

Received: 31 May 2020, Accepted: 04 August 2020, Online First: 05 September 2020, Published: 15 October 2020

\section{Edited by:}

Cem Vural

*Corresponding Author:

Nursel İkinci

nursel.ikinci@gmail.com

\section{ORCID ID:}

orcid.org/0000-0002-6435-5869

Key words:

Potamogetonaceae

Ecology

Macrophytes

Potamogeton praelongus

Potamogeton perfoliatus
Abstract: Clasping-leaved Potamogeton L. species growing in Turkey are P. praelongus Wulfen and $P$. perfoliatus L. There exists no detailed study about distribution, habitat requirements, and anatomical properties of the Turkish populations of the two species. Potamogeton perfoliatus is widespread throughout the country but $P$. praelongus was recorded only from a single locality. Therefore, $P$. praelongus is rare and endangered in Turkey. In this study, we recorded presence of $P$. perfoliatus in 54 wetlands based on examination of 86 herbarium specimens. Physical and chemical parameters of the water bodies where the two species occur were measured from 24 sites for $P$. perfoliatus and from one site for $P$. praelongus. According to our findings, $P$. praelongus grows in an alpine lake with oligotrophic, calcareous and alkaline water. Potamogeton perfoliatus occupies diverse habitats but prefers deep lentic water bodies with high $\mathrm{pH}$ and low salinity levels. Stem anatomy of the species were studied based on three individuals for P. praelongus and 35 individuals for $P$. perfoliatus. Morphological features of the species were also investigated and descriptions based on Turkish material were prepared. We provided the distinguishing anatomical and morphological characters between the species. Our anatomical findings showed that $P$. praelongus specimens have eight vascular bundles in contrast to previous reports on the species. Our results can be used for future monitoring of the two submerged Potamogeton species as we provide detailed information about their current distribution pattern and habitat features.

Özet: Türkiye'de yetişen gövdeyi saran yapraklı Potamogeton L. türleri P. praelongus Wulfen ve $P$. perfoliatus L. 'tur. Bugüne kadar bu türlerin dağılımı, habitat tercihleri ve anatomik özellikleriyle ilgili detaylı çalışmalar yoktur. Potamogeton perfoliatus ülke çapında yaygın bir türdür ancak $P$. praelongus sadece bir lokaliteden kaydedilmiştir. Bu nedenle $P$. praelongus Türkiye'de nadir ve tehdit altındadır. Bu çalışmada 86 herbaryum örneğine dayanarak $P$. perfoliatus'u 54 sulak alandan kaydettik. Türlerin yetiştiği suların fiziksel ve kimyasal parametreleri $P$. perfoliatus için 24 noktadan, $P$. praelongus için bir noktadan ölçülmüştür. Bulgularımıza göre, P. praelongus oligotrofik, kalkerli ve alkali alpin bir gölde yetişmektedir. Potamogeton perfoliatus çok farklı habitatlarda bulunmakla birlikte, yüksek $\mathrm{pH}$, düşük tuzluluk değerlerine sahip, derin ve durgun suları tercih etmektedir. Türlerin gövde anatomileri $P$. praelongus için 3 birey, $P$. perfoliatus için ise 35 bireyden örnek alınarak incelenmiştir. Türlerin morfolojik özellikleri de araştırılmış ve Türkiye'den toplanan materyallere dayalı olarak betimler hazırlanmıştır. Türler arasındaki ayırt edici anatomik ve morfolojik karakterler verilmiştir. Anatomi bulgularımız P. praelongus'un önceki bazı çalışmalara aykırı olarak sekiz iletim demetine sahip olduğunu göstermektedir. Bu çalışmada türlerin güncel dağılım ve habitat tercihleriyle ilgili sunduğumuz kapsamlı bulgular iki batık Potamogeton türünün gelecekte izlenmesinde faydalı olacaktır.

\section{Introduction}

Aquatic plants are primary producers and provide habitat and food to different organism groups, like algae, zooplankton, invertebrates, and different vertebrate taxa, such as fish and frogs (Bornette \& Puijalon 2011). Additionally, they are very important for establishment and maintenance of healthy ecosystems as they improve water quality due to their filtering capacity of excessive nutrients. They also affect water flow and sediment properties. Nutrient content of waters is very important for the diversity of macrophytes. It is known that the highest macrophyte diversity can be seen under moderate nutrient levels, and extreme nutrient levels favor only certain species. Under very low nutrient levels, stress tolerant species manage to survive and under eutrophic conditions, very competitive species grow in high densities and replace other species (Bornette \& Puijalon 2011). Therefore, macrophytes are very sensitive to habitat deteriorations mainly caused by anthropogenic 
alterations. Aquatic plants have faced an increasing extinction risk in the last decades due to eutrophication, water regime changes, pollution and introduction of invasive exotic species (Guo et al. 2019).

Potamogetonaceae is one of the largest aquatic plant families of the world with approximately 100 species growing in very diverse habitats. Potamogeton L., one of the most ecologically important genera of all aquatic plants (Haynes 1985), has the highest number of species in the family with species having solely floating or submerged leaves and species with both submerged and floating leaves (Wiegleb \& Kaplan 1998). The genus contains approximately 72 species and 99 hybrids (Kaplan et al. 2013). Taxonomy of the genus is quite difficult due to high number of hybrids and availability of limited reliable morphological characters (Wiegleb 1988). Additionally, Potamogeton species are known to exhibit extensive phenotypic plasticity, contributing to the difficulty of species delimitation.

First detailed taxonomical revision of Potamogeton was carried out by Ascherson and Graebner (1907) who divided Potamogeton into five sections and 13 subsections. Hagström (1916) published the most comprehensive study about Potamogeton including anatomical characters. Treatment of Hagström revealed five sections and 26 subsections. Potamogeton praelongus Wulfen (long-stalked or white-stemmed pondweed) belongs to the clasping leaved Potamogeton species group. Ascherson and Graebner (1907) evaluated $P$. praelongus and $P$. perfoliatus $\mathrm{L}$. with submerged and clasping-leaved species in the subsection Perfoliati Graebner of section Heterophylli K. Koch. However, Hagström (1916) separated the clasping-leaved species into two subsections: Subsection Perfoliati (Graebner) Hagström and subsection Praelongi Hagström. Haynes
(1985) followed Hagström's (1916) taxonomical treatment. More recently, Wiegleb (1988) recombined the two species in $P$. perfoliatus group.

Potamogetonaceae is the largest aquatic plant family in Turkey with 20 species and three hybrids belonging to five different genera (Uotila 1984, Aykurt et al. 2017, Bayındır 2018, Bayındır \& İkinci 2020a, 2020b). As the largest genus within the family, Potamogeton is represented by 14 species and three hybrid in Turkey. Potamogeton praelongus was included in the Flora of Turkey (Uotila 1984) based on a single record in the Flora of Caucasus (Grossheim 1928) from Kars province of north eastern Turkey. Since then, it could not be collected again and was thought to be extinct in Turkey. Although we visited several herbaria to examine Potamogeton species, we could not find any $P$. praelongus specimens collected from Turkey. However, during our fieldworks in 2016, we collected P. praelongus from a second locality in southern Turkey (Fig. 1, Bayındır 2018). According to Vöge (1992), world distribution of $P$. praelongus is Nordic, weakly suboceanic, and circumpolar. In Europe, its distribution extends from northern Scandinavia to south of France in the Alps and in Pyrenees. The species is also distributed in similar latitudes of Asia and North America. Even though the species is distributed in a wide geographical area, it is still a rare species (Prausová et al. 2011). It is considered as endangered in Switzerland and in Germany (Vöge 1992) and as critically endangered in the Czech Republic (Prausová et al. 2011). In the UK it is considered as near threatened and it is a protected species in France (Julve 2017). Prausová et al. (2014) stated that P. praelongus is endangered in all Central Europe. However, IUCN assessed it as Least Concern (Lansdown 2014).

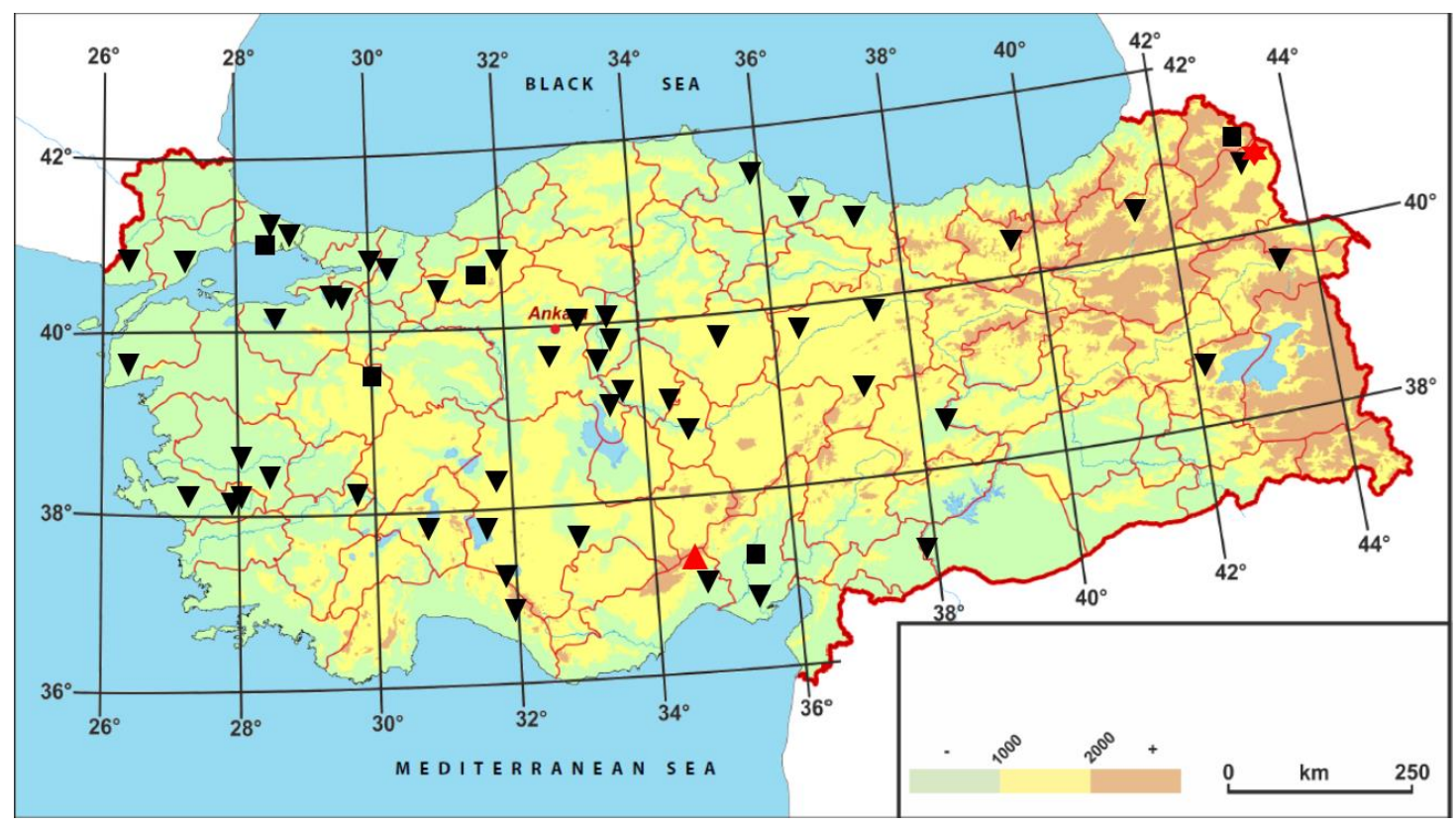

Fig. 1. Distribution map of P. praelongus ( $\mathbf{\Delta}$ current location, $\mathbf{t}$ historical location) and P. perfoliatus ( $\mathbf{\nabla}$ according to Bayindır (2018) and new collection sites added with this study) in Turkey. Revised from Bayındır (2018). 
Potamogeton perfoliatus is the other clasping leaved species in Turkey and is widespread throughout the country except the southeastern Anatolia. This region of Turkey has a hot and semi-arid climate with semi-arid steppe vegetation different from the rest of the country (Ergüner et al. 2019). According to Gupta \& Lansdown (2013), P. perfoliatus can be considered a cosmopolitan species as it has a distribution throughout Eurasia (from the Mediterranean to northern Scandinavia and Iceland, Siberia), in North America and Greenland, in North and Central Africa and in Australia. It is assessed as Least Concern (LC) by IUCN (Gupta \& Lansdown 2013). Haynes (1985) stated that $P$. perfoliatus is a morphologically very variable species. Therefore, several subspecific categories were defined for the species. However, these variations are mainly on vegetative characters and do not require distinct taxonomic divisions (Haynes 1985).

Studies on $P$. perfoliatus are mainly restricted to floristic records and altitudinal distribution analysis (Bayındır 2018, İkinci \& Bayındır 2019, İkinci \& Bayındır 2020) and there exist no study about the anatomy and habitat requirements of the species in Turkey. Therefore, we aimed in this study i) to provide detailed information about the geographical distribution of the clasping-leaved Potamogeton species in Turkey, ii) to determine the habitat features of the species including water chemistry and other environmental parameters, iii) to prepare new morphological descriptions of the species based on Turkish material and v) to provide morphological and anatomical comparisons between $P$. praelongus and $P$. perfoliatus.

\section{Materials and Methods}

\section{$\underline{\text { Plant Materials }}$}

The plant material was collected from the field between 2014 and 2017 as a part the project on the revision of Potamogetonaceae family in Turkey. Entire plants were taken from fresh material in the field and preserved in $70 \%$ alcohol solution for anatomical studies which are stored at Bolu Abant İzzet Baysal University Herbarium (AIBU) (Table 1). Herbarium specimens were also prepared for each sample and stored in AIBU. Additionally, the following herbaria were visited to study the Potamogeton species: ANK, ISTE, ISTF, HUB, ISTO, ADA, K, E, L, LINN, VANF (acronyms according to Thiers 2016).

\section{Environmental Parameter Measurements}

Physical and chemical parameters of the water bodies where the two Potamogeton species were sampled were measured from 25 different wetlands during the growth season of the plants in 2016 and 2017. We sampled the plants and physical and chemical properties of the water from the littoral zone of water bodies. In deeper sites, we gathered macrophytes with the help of a rake. Single measurements were made per site for physical and chemical variables. We measured seven environmental variables with a portable multi probe YSI-Professional Plus in situ. The environmental variables were dissolved oxygen concentration (DO, $\mathrm{mg} \mathrm{l}^{-1}$ ), water temperature $\left(\mathrm{Tw},{ }^{\circ} \mathrm{C}\right)$, electrical conductivity $\left(\mathrm{EC}, \mu \mathrm{S} \mathrm{cm}^{-1}\right), \mathrm{pH}$, total dissolved solids (TDS, $\mathrm{g} \mathrm{l}^{-1}$ ), salinity (ppt) and ammonium $\left(\mathrm{mg} \mathrm{l}^{-1}\right)$. The altitudes of the sampling sites were recorded by using Magellan eXplorist 610. Environmental variables and abundances were measured at random locations where the species were collected. We estimated abundance visually and recorded the percentage area covered by each species. Abundance percentage measurements were based on ACFOR scale [abundant (>80\% cover), common (61-80\%), frequent (31-60\%), occasional (5-30\%), and rare $(<5 \%)$ ] (Crisp \& Southward 1958, Dunham et al. 2018). The estimated values were converted to numerical scores from 1 to 5 . We also recorded the accompanying aquatic plant species (Table 1).

Table 1. List of our collections for P. perfoliatus with information about other accompanying Potamogetonaceae species.

\begin{tabular}{|c|c|c|c|c|c|c|c|c|c|c|c|c|c|c|c|c|}
\hline Locality & Coordinates & $\begin{array}{l}\text { Altitude } \\
\text { (m) }\end{array}$ & Date & $\begin{array}{l}\text { Anatomy } \\
\text { material }\end{array}$ & 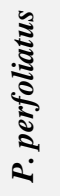 & 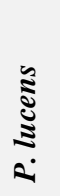 & 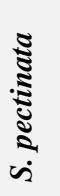 & 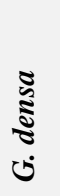 & 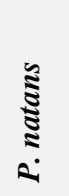 & 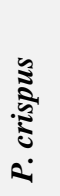 & 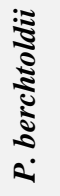 & 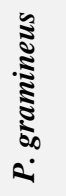 & 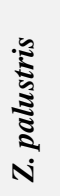 & 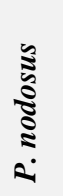 & 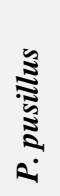 & 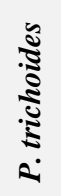 \\
\hline $\begin{array}{l}\text { *Adana: Seyhan } \\
\text { Dam Lake }\end{array}$ & $\begin{array}{l}37.04921 \mathrm{~N} \\
35.31672 \mathrm{E}\end{array}$ & 59 & 27.vi.2016 & $\sqrt{ }$ & + & + & + & & & & & & & & & \\
\hline $\begin{array}{l}\text { Antalya: } \\
\text { Gündoğmuş, Lake } \\
\text { Eğil }\end{array}$ & $\begin{array}{l}36.93217 \mathrm{~N} \\
32.19954 \mathrm{E}\end{array}$ & 2078 & 25.vi.2016 & $\sqrt{ }$ & + & + & & + & + & & & & & & & \\
\hline $\begin{array}{l}\text { * Ardahan: } \\
\text { Güvenocak, Çıldır } \\
\text { Lake }\end{array}$ & $\begin{array}{l}41.09930 \mathrm{~N} \\
43.24034 \mathrm{E}\end{array}$ & 1959 & 11.vii.2017 & - & + & & + & & & + & & & & & & \\
\hline * Bolu: Gölköy Lake & $\begin{array}{l}40.42^{\prime} 06 \mathrm{~N} \\
31.31^{\prime} 09 \mathrm{E}\end{array}$ & 770 & $\begin{array}{l}\text { 02.ix.2014 } \\
\text { 14.vii.2014 }\end{array}$ & - & + & + & + & + & & + & + & + & + & & & \\
\hline $\begin{array}{l}\text { Bolu: Yeniçağa } \\
\text { Lake, S. of the lake }\end{array}$ & $\begin{array}{c}40.77353 \mathrm{~N} \\
32.02366 \mathrm{E}\end{array}$ & 989 & 13.vii.2015 & $\sqrt{ }$ & + & & & & & & + & & & & & \\
\hline
\end{tabular}




\begin{tabular}{|c|c|c|c|c|c|c|c|c|c|c|c|c|c|c|c|c|}
\hline Locality & Coordinates & $\begin{array}{l}\text { Altitude } \\
\text { (m) }\end{array}$ & Date & $\begin{array}{c}\text { Anatomy } \\
\text { material }\end{array}$ & 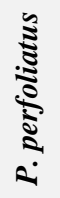 & $\begin{array}{c}\vdots \\
\vdots \\
\vdots \\
2\end{array}$ & 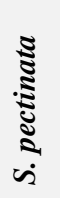 & 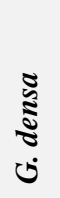 & 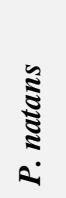 & 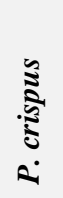 & 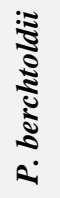 & 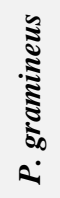 & 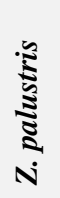 & 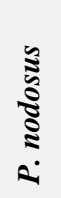 & 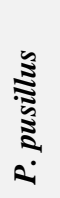 & 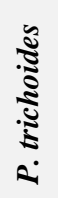 \\
\hline $\begin{array}{l}\text { Bursa: İznik Lake, } \\
\text { near wharf }\end{array}$ & $\begin{array}{l}40.47785 \mathrm{~N} \\
29.66242 \mathrm{E}\end{array}$ & 85 & 29.vii.2015 & $\sqrt{ }$ & + & & & & & & & & & & & \\
\hline Bursa: İznik Lake & $\begin{array}{l}40.44677 \mathrm{~N} \\
29.71397 \mathrm{E}\end{array}$ & 87 & 29.vii.2015 & $\sqrt{ }$ & + & & + & & & + & & & + & & & \\
\hline $\begin{array}{l}\text { Bursa: İznik Lake, } \\
\text { Boyalıca }\end{array}$ & $\begin{array}{l}40.48302 \mathrm{~N} \\
29.56499 \mathrm{E}\end{array}$ & 83 & 29.vii.2015 & $\sqrt{ }$ & + & & & & & & & & & & & \\
\hline $\begin{array}{l}\text { Bursa: Ulubat Lake, } \\
\text { bird watching pier }\end{array}$ & $\begin{array}{l}40.19893 \mathrm{~N} \\
28.44291 \mathrm{E}\end{array}$ & 1 & 30.vii.2015 & $\sqrt{ }$ & + & & & & & + & & & & & & \\
\hline $\begin{array}{l}\text { Denizli: Çivril, Işıklı } \\
\text { Lake, entrance to } \\
\text { Beydilli }\end{array}$ & $\begin{array}{l}38.25761 \mathrm{~N} \\
29.93300 \mathrm{E}\end{array}$ & 827 & 19.vi.2016 & $\sqrt{ }$ & + & + & + & & & + & & & & & & \\
\hline $\begin{array}{l}\text { Edirne: Enez, W. of } \\
\text { Gala Lake }\end{array}$ & $\begin{array}{l}40.75159 \mathrm{~N} \\
26.16873 \mathrm{E}\end{array}$ & 34 & 12.vi.2016 & $\sqrt{ }$ & + & & + & & & + & & & & + & & \\
\hline $\begin{array}{l}\text { Erzurum: Uzundere, } \\
2 \mathrm{~km} \text { to Tortum } \\
\text { Dam }\end{array}$ & $\begin{array}{l}41.57944 \mathrm{~N} \\
41.60204 \mathrm{E}\end{array}$ & 1018 & 19.viii.2016 & $\sqrt{ }$ & + & & + & & & & + & & & & & \\
\hline $\begin{array}{l}\text { Gümüşhane: } \\
\text { Erzincan-Bayburt } \\
\text { Road, Salyazı Dam }\end{array}$ & $\begin{array}{l}40.24449 \mathrm{~N} \\
39.81048 \mathrm{E}\end{array}$ & 1684 & 20.viii.2016 & $\sqrt{ }$ & + & & + & & & & & + & & & & \\
\hline $\begin{array}{l}\text { İstanbul: } \\
\text { Arnavutköy, Terkos } \\
\text { Lake }\end{array}$ & $\begin{array}{l}41.37284 \mathrm{~N} \\
28.56756 \mathrm{E}\end{array}$ & 2 & 10.viii.2016 & $\sqrt{ }$ & + & + & & & & & & & & & + & \\
\hline $\begin{array}{l}\text { * İstanbul: } \\
\text { Büyükçekmece, } \\
\text { Büyükçekmece Lake }\end{array}$ & $\begin{array}{l}41.06745 \mathrm{~N} \\
28.57156 \mathrm{E}\end{array}$ & -1 & 10.vi.2016 & $\sqrt{ }$ & + & & & & & & & & & & + & \\
\hline $\begin{array}{l}\text { İstanbul: Eyüp, } \\
\text { Göktürk, Göktürk } \\
\text { Dam Lake }\end{array}$ & $\begin{array}{l}41.19319 \mathrm{~N} \\
28.87544 \mathrm{E}\end{array}$ & 41 & 10.vi.2016 & $\sqrt{ }$ & + & & & & + & + & + & & & & & + \\
\hline $\begin{array}{l}\text { İzmir: Torbalı, } \\
\text { Torbalı Dam Lake }\end{array}$ & $\begin{array}{l}38.17277 \mathrm{~N} \\
27.16007 \mathrm{E}\end{array}$ & 63 & 20.vi.2016 & $\sqrt{ }$ & + & & & & & + & + & & & & & \\
\hline $\begin{array}{l}\text { Kars: Doğruyol, } \\
\text { Çıldır Lake }\end{array}$ & $\begin{array}{l}41.06916 \mathrm{~N} \\
43.32759 \mathrm{E}\end{array}$ & 1960 & 11.viii.2017 & - & + & & + & & & & & & & & + & \\
\hline $\begin{array}{l}\text { Kırıkkale: Ankara } \\
\text { border, Kızılırmak } \\
\text { River }\end{array}$ & $\begin{array}{l}39.93968 \mathrm{~N} \\
33.41290 \mathrm{E}\end{array}$ & 661 & 22.viii.2016 & - & + & & + & & & & & & + & & & \\
\hline $\begin{array}{l}\text { Kırkkkale: Keskin, } \\
\text { Köprüköy, } \\
\text { Kapulukaya Dam }\end{array}$ & $\begin{array}{l}39.57404 \mathrm{~N} \\
33.43205 \mathrm{E}\end{array}$ & 719 & 05.ix.2016 & $\sqrt{ }$ & + & & & + & & & & & + & & & \\
\hline $\begin{array}{l}\text { Kırşehir: Mucur, } \\
\text { near Karkın Village, } \\
\text { Kargın Dam Lake }\end{array}$ & $\begin{array}{l}39.00823 \mathrm{~N} \\
34.48361 \mathrm{E}\end{array}$ & 1086 & 05.ix.2016 & $\sqrt{ }$ & + & & + & & & + & + & & & & & \\
\hline $\begin{array}{l}\text { Konya: Bozkır, } \\
\text { Dipsizgöl }\end{array}$ & $\begin{array}{l}37.10161 \mathrm{~N} \\
32.04097 \mathrm{E}\end{array}$ & 1687 & 08.ix.2016 & $\sqrt{ }$ & + & & & & & + & & & & + & & \\
\hline $\begin{array}{l}\text { Konya: Ilgin, } \\
\text { Çavuşçu Lake }\end{array}$ & $\begin{array}{l}38.37327 \mathrm{~N} \\
31.89334 \mathrm{E}\end{array}$ & 1016 & 08.ix.2016 & - & + & & & & & & & + & & & & \\
\hline $\begin{array}{l}\text { Konya: Çumra, } \\
\text { Türkmenkarahüyük } \\
\text { Village }\end{array}$ & $\begin{array}{l}37.61108 \mathrm{~N} \\
33.02601 \mathrm{E}\end{array}$ & 992 & 08.ix.2016 & $\sqrt{ }$ & + & & & & & & & & & & & \\
\hline $\begin{array}{l}\text { * Kütahya: } \\
\text { Eskişehir-Kütahya } \\
\text { Road, Sofça Village, } \\
\text { Porsuk Dam }\end{array}$ & $\begin{array}{l}39.60567 \mathrm{~N} \\
30.14925 \mathrm{E}\end{array}$ & 895 & 18.vi.2016 & $\sqrt{ }$ & + & & + & & & & + & & + & & & \\
\hline
\end{tabular}




\begin{tabular}{|c|c|c|c|c|c|c|c|c|c|c|c|c|c|c|c|c|}
\hline Locality & Coordinates & $\begin{array}{l}\text { Altitude } \\
\text { (m) }\end{array}$ & Date & $\begin{array}{c}\text { Anatomy } \\
\text { material }\end{array}$ & 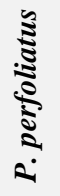 & 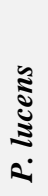 & 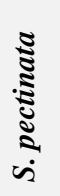 & 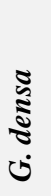 & 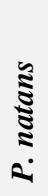 & 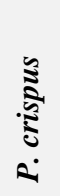 & 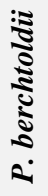 & 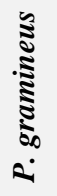 & 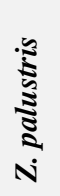 & 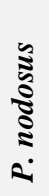 & 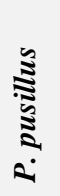 & 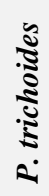 \\
\hline $\begin{array}{l}\text { Mersin: Tarsus Dam } \\
\text { Lake }\end{array}$ & $\begin{array}{l}36.95359 \mathrm{~N}, \\
34.89384 \mathrm{E}\end{array}$ & 35 & 27.vi.2016 & $\sqrt{ }$ & + & & + & & & + & & & & + & & \\
\hline $\begin{array}{l}\text { Ordu: Fatsa, } \\
\text { Sefaköy, Gaga Lake }\end{array}$ & $\begin{array}{l}40.75159 \mathrm{~N}, \\
26.16873 \mathrm{E}\end{array}$ & 67 & 15.viii.2016 & $\sqrt{ }$ & + & & & & & & & & & + & & \\
\hline $\begin{array}{l}\text { Sakarya: Sapanca } \\
\text { Lake, Eşme Village } \\
\text { shores }\end{array}$ & $\begin{array}{c}40.73328 \mathrm{~N} \\
30.23521 \mathrm{E}\end{array}$ & 26 & $\begin{array}{l}\text { 29.vii.2015 } \\
\text { 15.viii. } 2015\end{array}$ & $\sqrt{ }$ & + & + & & & & & & & & + & & + \\
\hline $\begin{array}{l}\text { Samsun: Ayvacık, } \\
\text { Suat Uğurlu Dam } \\
\text { Lake }\end{array}$ & $\begin{array}{c}41.07370 \mathrm{~N}, \\
36.66831 \mathrm{E}\end{array}$ & 53 & 14.viii.2016 & $\sqrt{ }$ & + & & + & & & & & & & & & \\
\hline $\begin{array}{l}\text { Samsun: Delta } \\
\text { Kızılırmak, bird } \\
\text { sanctuary }\end{array}$ & $\begin{array}{l}41.67075 \mathrm{~N} \\
36.03464 \mathrm{E}\end{array}$ & 3 & 14.viii.2016 & $\sqrt{ }$ & + & & + & & & & & & & + & & \\
\hline $\begin{array}{l}\text { Sivas: Hafik, Hafik } \\
\text { Lake }\end{array}$ & $\begin{array}{l}39.87247 \mathrm{~N}, \\
37.38256 \mathrm{E}\end{array}$ & 1269 & 21.viii.2016 & $\sqrt{ }$ & + & & + & & & & & & & & & \\
\hline $\begin{array}{l}\text { Sivas: Halkaçayır } \\
\text { Village, Dam Lake }\end{array}$ & $\begin{array}{l}39.80974 \mathrm{~N}, \\
36.34908 \mathrm{E}\end{array}$ & 1372 & 21.viii.2016 & $\sqrt{ }$ & + & & & & & & & & & & & + \\
\hline $\begin{array}{l}\text { Tekirdağ: Yazır } \\
\text { Dam Lake }\end{array}$ & $\begin{array}{l}40.92561 \mathrm{~N} \\
27.40341 \mathrm{E}\end{array}$ & 59 & 12.vi.2016 & $\sqrt{ }$ & + & & + & & & + & & & & + & & \\
\hline $\begin{array}{l}\text { Yozgat: Entrance to } \\
\text { Sorgun, Mükremin } \\
\text { Pond }\end{array}$ & $\begin{array}{l}39.80372 \mathrm{~N} \\
35.21713 \mathrm{E}\end{array}$ & 1076 & 21.viii.2016 & $\sqrt{ }$ & + & & + & & & & & & & & & \\
\hline Total (34 wetlands) & & & & 29 & 36 & 6 & 18 & 3 & 2 & 12 & 7 & 3 & 5 & 7 & 3 & 3 \\
\hline
\end{tabular}

* New wetlands added to the list in Bayındir (2018).

\section{Anatomical studies}

Plant samples were taken to represent all parts of the plants for morphological diagnosis and samples preserved in $70 \%$ alcohol solution were used for subsequent anatomical studies. Stem anatomy samples were prepared from three individuals for $P$. praelongus and from 35 individuals for $P$. perfoliatus. The internode areas of stems of the specimens were cut about $0.05 \mathrm{~mm}$ thick with the aid of razor blades. Samples were put into safranin or toluidine blue dye and transferred to distilled water. Stem fragments were examined under the light microscope at 4x, 10x and 40x magnifications. The stele types, shape of endodermal cells, pseudohypodermis, presence of subepidermal bundles and interlacunar bundles in the cortex were determined and photographed.

\section{Results}

\section{Potamogeton praelongus Wulfen}

\section{Description}

Stem slightly branched or unbranched with rhizome; terete; clearly zig-zag shaped; mostly white or pale green, up to $200 \mathrm{~cm}$ in length. Leaves sessile, all submerged, alternate, lax, mostly undulate or entire, pale green to olive green, lanceolate to broadly lanceolate, broadly ovate elliptic, 11-17 veined, midrib without lacunae, 52 $171 \times 14-27 \mathrm{~mm}$, margin entire, at base cuneate and semiamplexicaule, at apex clearly cucullate, (splitting when pressed), obtuse. Stipules persistent, conspicuous, convolute, free from blade, white, 17-42 mm, fibrous.

\section{Locality}

TURKEY, Mersin, Çamliyayla, Darboğaz, near Summit Medetsiz, Karagöl Lake, 2591 m, 37.404261 N, 34.559753 E, 07 September 2016, N. Bayındır 1360 (AIBU 12735) (Fig. 1).

Table 2. Water chemistry measurements for $P$. praelongus (see materials and methods for abbreviations).

\begin{tabular}{|c|c|c|c|c|c|c|}
\hline $\begin{array}{l}\text { Tw } \\
\left({ }^{\circ} \mathbf{C}\right)\end{array}$ & pH & $\underset{\left(\mathrm{mg} \mathrm{l}^{-1}\right)}{\text { DO }}$ & $\begin{array}{c}\text { EC } \\
\left(\mu \mathrm{S} \mathrm{cm}^{-1}\right)\end{array}$ & $\begin{array}{l}\text { TDS } \\
\left(\mathrm{g} \mathrm{l}^{-1}\right)\end{array}$ & $\begin{array}{c}\text { Salinity } \\
\text { (ppt) }\end{array}$ & $\underset{\left(\mathrm{mg} \mathrm{l}^{-1}\right)}{\operatorname{Ammonium}}$ \\
\hline 17.9 & 7.86 & 5.92 & 165.9 & 0.1248 & 0.09 & 0.02 \\
\hline
\end{tabular}

\section{Ecological notes}

Potamogeton praelongus was collected from an oligotrophic, calcareous, snow-melting lake (Karagöl Lake) with alkaline water (Table 2). In addition to $P$. praelongus, P. natans L., Stuckenia filiformis (Persoon) 


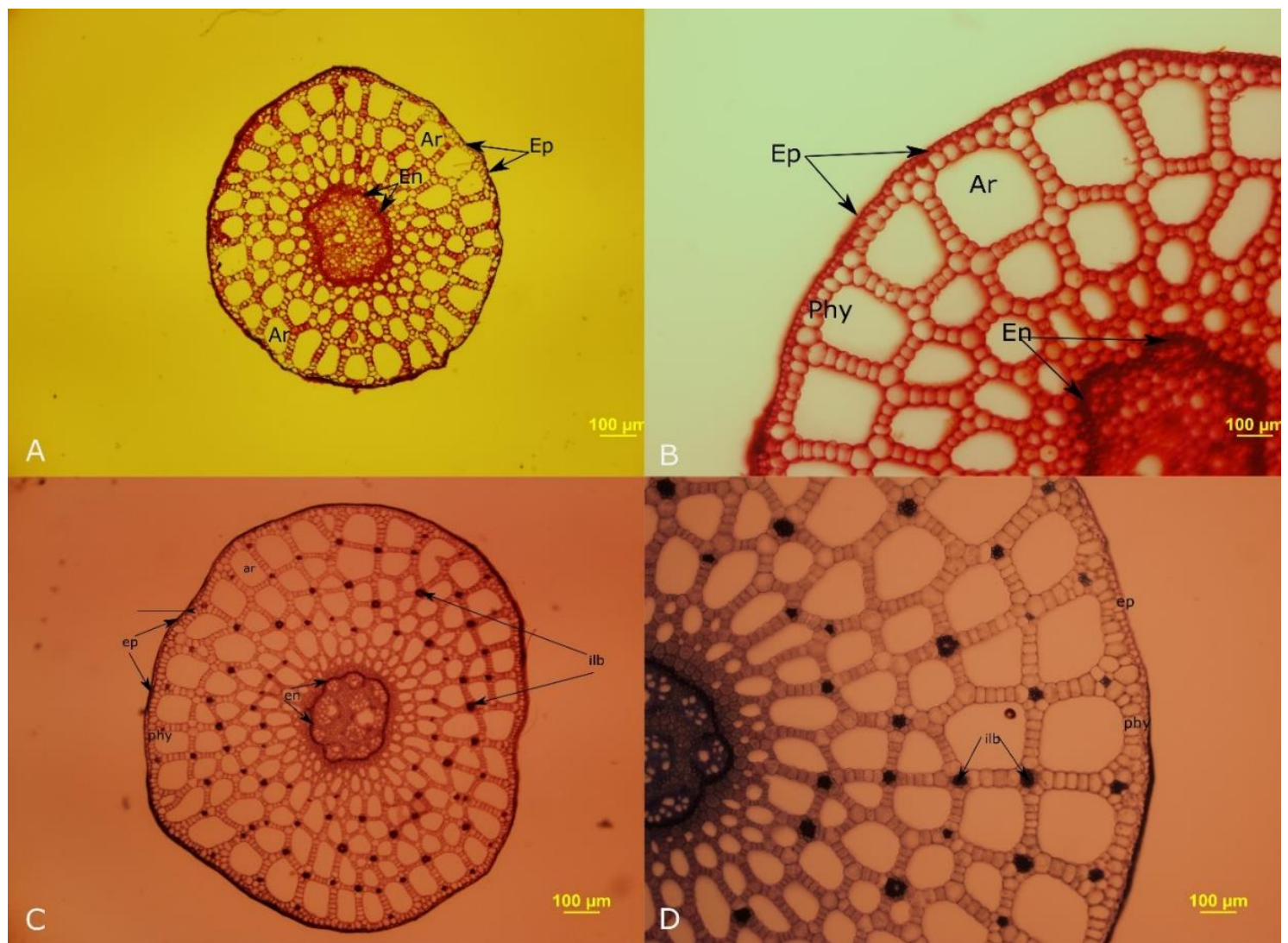

Fig. 2. Stem anatomy of $P$. perfoliatus (A, B) and P. praelongus (C, D). General view (A, C) $(4 \times)$, Epidermis and cortex (B, D) $(10 \times)$, (Ar: aerenchyma, En: endodermis, Ep: epidermis, Ilb: interlacunar bundle, Phy: pseudohypodermis).

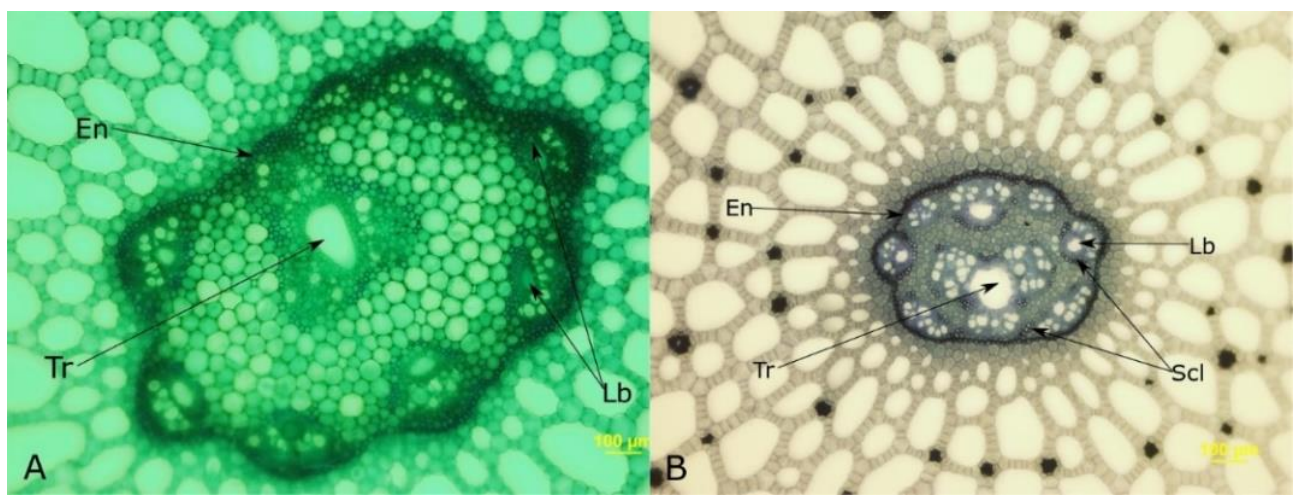

Fig. 3. Stele of $P$. perfoliatus (A) and P. praelongus (B) (10×), (En: endodermis, Lb: lateral bundle, Scl: sclerenchyma, Tr: trio-bundle).

Börner, Persicaria amphibia (L.) Gray were growing in the lake. Since the species is currently known only from this site in Turkey, we have single measurements of the physical and chemical parameters of the water body where the species was sampled.

\section{Anatomy}

Our anatomical findings for $P$. praelongus and $P$. perfoliatus are shown in Figs 2-3 and Table 3.

\section{Potamogeton perfoliatus $L$.}

\section{Description}

Stem slightly unbranched or branched at the top with rhizome; terete; dark green, pale green, whitish or brownish upto $200 \mathrm{~cm}$ length; without turion. Leaves sessile, all submerged, alternate, undulate or entire, usually olive-green, rarely dark green, rarely leaves with rusted colour, broadly lanceolate, broadly ovate or close to rounded, 5-25 veined, midrib without lacunae, 17-61 $\times$ 12-34 mm, margin slightly denticulate, at base cordate and amplexicaul, at apex mostly entire or slightly cucullate, obtuse, rounded or acute. Stipule free from blade, convolute, mostly deciduous rarely persistent, ca. $14 \mathrm{~mm}$ long, nonfibrous. Inflorescence spike, terminal, sometimes axillary, cylindrical, 19-27 mm long, continuous; flowers dense. Carpels 4. Fruits sessile, obovate, $2.5-3.5 \times 1.8-2.25 \mathrm{~mm}$; beak short, dark green to blackish, 0.3-0.5 mm long. 
Table 3. Comparison of anatomical characters of $P$. praelongus and $P$. perfoliatus based on our collections.

\begin{tabular}{lccc}
\hline \hline \multicolumn{1}{c}{ Character } & P. praelongus & P. perfoliatus \\
\hline \hline $\begin{array}{l}\text { Stele types } \\
\begin{array}{l}\text { Shape of } \\
\text { endodermal cells }\end{array}\end{array}$ & Trio type & Trio type \\
$\begin{array}{l}\text { Pseudohypodermis } \\
\begin{array}{l}\text { Presence of } \\
\text { subepidermal } \\
\text { bundles }\end{array}\end{array}$ & 1 seriate & O type \\
$\begin{array}{l}\text { Presence of } \\
\text { interlacunar } \\
\text { bundles in the } \\
\text { cortex }\end{array}$ & Present & $\begin{array}{c}\text { Absent/rarely } \\
\text { present }\end{array}$ \\
\hline \hline
\end{tabular}

\section{Additional herbarium specimens examined}

The information of the examined specimens at different herbaria are given in the below list. The list was organized in alphabetical order based on the names of the Turkish provinces (in bold). There are more than one herbarium specimens from the same wetland. The herbarium acronyms are in parenthesis.

Examined material: Adana: Yenice irrigation, main canal, G. Altınayar (DSI 1988). Ağrı: Balık Lake shores, 2210 m, 12 vi 1979, A. Baytop, B. Çubukçu (ISTE 42741). Balık Lake, 2261 m, 29 ix 2014, M.M. Uma, M. Kaya (AIBU). Suluçem (Musun), S. end of Balık Lake, $2300 \mathrm{~m}$, shallow water, 23 vii 1967, P.H. Davis 47279 (K). Ankara: Bala, Kesikköprü Dam Lake, 790 m, 12 vii 2014, A.E. Yaprak, S.T. Körüklü, İ. Başköse, A. Güleryüz (AIBU, ANK). Elmadağ, 14 vii 1939, B. Kasaplıil (ISTF 572). Eymir Lake, 08 vii 1933, W. Kotte (ANK). Bitlis: Nazik Lake shores, submerged, ca. 1870 m, 31 viii 1993, L. Behçet 4691 (VANF). Bolu: Abant Lake, 08 viii 1970, A. and T. Baytop (ISTE 18397). Yeniçağa Lake, near Gerede, 03 x 1961, A. and T. Baytop (ISTE 6882). Bursa: İznik, İznik Lake, ca. 85 m, 20 vii 1981, P. Uotila (EGE 24903). Çanakkale: Ayvacık, Creek Geme, 243 m, 03 ix 2014, E. Cabi, M.M. Uma, N. Albayrak (AIBU). Denizli: Çivril, W. of Işıklı Lake, Buca Village, ca. 850 m, 15 vi 1981, E. Leblebici (EGE 26217). Edirne: Enez, Gala Lake, G. Altınayar (DSI 1988). Isparta: N. of Eğirdir Lake, Hayran, ca. 940 m, 09 vii 1980, E. Leblebici (EGE 26841). İstanbul: Terkos Village, in the lake, 29 ix 1969, A. Baytop (E 00330333). Çatalca, Terkos Lake, 01 ix 1943, B. Kasaplıgil (ISTF 5282). Kurtköy, Terkos Lake shore, 04 x 1967, A. Baytop, G. Atila (ISTE 12146). Terkos Village, in the lake, 29 ix 1967, A. Baytop (ISTE). Terkos Lake, 29 vii 1952, A. Baytop (ISTE 2835). Terkos Lake, 02 vii 1969, A. Baytop (ISTE 15527). Terkos Lake, 17 vii 1970, A. Baytop (ISTE 18182). İzmir: Ödemiş, Bozdağ, Gölcük, 04 vii 1966, C. Regel (EGE 24864). Ödemiş, Gölcük, submerged, 07 vii 1962, C. Regel (EGE1851). Ödemiş, road to Gölcük, 08 vi 1946, A. Heilbronn, M. Başarman (ISTF). Ödemiş, Bozdağ Village, Kırkoluklar fountain, ca. 1000 m, 09 vi 1980, E. Leblebici (EGE 2680). Kars: Ç1ldır-Kars road, Lake Çıldır shore, 20 viii 1975, A. Baytop, E. Tuzlacı
(ISTE 33416). Kırıkkale: Before Kizılırmak-Balaban stream mixing site, 678 m, 27 ix 2014, A.E. Yaprak, S.T. Körüklü, İ. Başköse, A. Güleryüz (AIBU, ANK). Kurklareli: City centre, Koyunbaba Village, Koyunbaba spring, submerged, 170 m, 24 v 1994, E. Üzen (ISTF). Kırşehir: Hirfanlı Dam, 875 m, 12 vii 2014, A.E. Yaprak, S.T. Körüklü, İ. Başköse, A. Güleryüz (AIBU, ANK). Kocaeli: Saracoğlu Motel at the Lake Sapanca, floating on the shore of the lake, 40 m, 01 ix 1972, P. Uotila 20153 (E). Sakarya: E. shores of Lake, near Arifiye, 04 vii 1976, A. Baytop, K. Alpınar (ISTE35123). N. shore of Sapanca Lake, 24 viii 1973, H. Güner (EGE12774). Sapanca Lake, 50 m, edge of lake, 30 vi 1962, P.H. Davis 36207, M.J.E. Coode (K). Konya: Akşehir, E. of Ilgın Village, Gedikören Village, ca. $1000 \mathrm{~m}, 08$ vii 1980, E. Leblebici (EGE 26830). Ilgın, Çavuşçu Lake, submerged, 08 viii 1992, A.A. Dönmez, N. Emir (GAZI, HUB). Between BeyşehirŞarkıkaraağaç, $12 \mathrm{~km}$ to Beyşehir, in water, $1100 \mathrm{~m}, 03$ viii 1978, A. Baytop, E. Tuzlacı (ISTE41432). Beyşehir, Çiftlikköy, shores, 06 vii 2014, T. Körüklü (AIBU 12345). Beyşehir, 01 vi 1949, İ. Baykal (ISTF). Beyşehir (Isawia), Hoynan, in the lake, 05 viii 1949, P.H. Davis 16113 (K). Çumra irrigation canal, Yeni İsmil reserve irrigation canal, G. Altınayar (DSI 1988). Malatya: Karakaya Dam, 690 m, 19 vii 2014, M.M. Uma, N. Albayrak (AIBU). Manisa: Adala irrigation canal to Marmara Lake, G. Altınayar (DSI 1988). Salihli, N. of Gölmarmara, Sazköyü, ca. 90 m, 03 vi 1981, E. Leblebici (EGE26144). Nevşehir: KızılırmakGülşehir Bridge, 888 m, 13 vii 2014, A.E. Yaprak, S.T. Körüklü, İ. Başköse, A. Güleryüz (AIBU, ANK). Ordu: Kaga Göl above Fatsa, 250 m, deep warm lake, 21 vii 1965, C. Tobey 1312 (E). Samsun: Bafra, Kızılırmak, 8 m, 08 vii 2014, A.E. Yaprak, S.T. Körüklü, İ. Başköse, A. Güleryüz (AIBU, ANK). Sivas: Kangal, Üçöz Dam Lake, 1573 m, 19 vii 2014, M.M. Uma, N.Albayrak (AIBU). Şanlıurfa: Birecik Dam Lake, 392 m, 24 vii 2014, M.M. Uma, N. Albayrak (AIBU). Van: Between Doğubeyazıt and Taşlıçay, Balık Lake, ca. 2450 m, 11 viii 1985, L. Behçet (VANF).

\section{Ecological notes}

Measurements of the physical and chemical parameters of the water bodies for $P$. perfoliatus were performed in 24 wetlands (Table 4). Unlike $P$. praelongus, $P$. perfoliatus is widespread and occurs in diverse habitats. The $\mathrm{pH}$ range of the water it occurs ranges from 7.52 to 9.01 . Based on our collections from 34 wetlands, $P$. perfoliatus occurs together with other Potamogetonaceae species in 31 of these wetlands (Table $1)$. According to these findings, $P$. perfoliatus co-occurs in 18 wetlands with Stuckenia pectinata (L.) Börner, in 12 wetlands with $P$. crispus L., in 7 wetlands with $P$. nodosus Poir. and $P$. berchtoldii Fieber, in 6 wetlands with $P$. lucens L., and in 5 wetlands with Zannichelia palustris L., Groenlandia densa (L.) Fourr., P. gramineus L., $P$. pusillus L. and P. trichoides Cham. \& Schltdl. were found to accompany $P$. perfoliatus in 3 wetlands. Potamogeton natans was found to grow together with $P$. perfoliatus at 2 sites and in 3 sites $P$. perfoliatus was the only species growing. 
Table 4. Physical and chemical measurements for P. perfoliatus (NB stands for collector N. Bayındır. See materials and methods for additional abbreviations). Detailed information about each voucher is given in Table 1.

\begin{tabular}{|c|c|c|c|c|c|c|c|c|c|}
\hline Voucher & $\begin{array}{l}\text { Sampling } \\
\text { date }\end{array}$ & $\begin{array}{l}\text { Tw } \\
\left({ }^{\circ} \mathbf{C}\right)\end{array}$ & pH & $\begin{array}{c}\text { DO } \\
\left(\mathrm{mg} \mathrm{l}^{-1}\right)\end{array}$ & $\begin{array}{c}\mathrm{EC} \\
\mu \mathrm{S} \mathrm{cm}^{-1}\end{array}$ & $\begin{array}{c}\text { TDS } \\
\left(\mathrm{g} \mathrm{l}^{-1}\right)\end{array}$ & $\begin{array}{c}\text { Salinity } \\
\text { (ppt) }\end{array}$ & Ammonium (mg l-1) & Abundance \\
\hline NB 1178 & 10.6 .2016 & 22.1 & 8.28 & 5.64 & 427.8 & 0.2944 & 0.22 & 0.50 & 3 \\
\hline NB 1182 & 10.6 .2016 & 23.6 & 8.49 & 8.48 & 309.9 & 0.2067 & 0.15 & 0.14 & 2 \\
\hline NB 1185 & 10.6 .2016 & 25.9 & 8.31 & 10.56 & 710.0 & 0.4615 & 0.34 & 0.38 & 4 \\
\hline NB 1208 & 12.6 .2016 & 27.6 & 7.53 & 3.98 & 2253 & 1.4040 & 1.10 & 1.11 & 1 \\
\hline NB 1216 & 18.6.2016 & 24.7 & 8.86 & 9.03 & 550.0 & 0.2675 & 0.27 & 0.72 & 3 \\
\hline NB 1228 & 19.6.2016 & 28.0 & 8.99 & 5.83 & 317.7 & 0.1911 & 0.12 & 0.32 & 4 \\
\hline NB 1235 & 20.6 .2016 & 31.2 & 8.82 & 12.31 & 369.3 & 0.2080 & 0.15 & 0.42 & 4 \\
\hline NB 1261 & 25.6 .2016 & 23.1 & 8.09 & 6.95 & 241.3 & 0.1631 & 0.12 & 0.26 & 4 \\
\hline NB 1268 & 27.6.2016 & 18.4 & 7.57 & 8.99 & 259.0 & 0.1924 & 0.14 & 0.39 & 3 \\
\hline NB 1273 & 27.6 .2016 & 29.5 & 8.23 & 6.35 & 455.2 & 0.2723 & 0.20 & 0.21 & 2 \\
\hline NB 1293 & 14.8 .2016 & 23.9 & 7.73 & 7.53 & 1977 & 1.3130 & 1.03 & 0.59 & 2 \\
\hline NB 1296 & 14.8 .2016 & 24.1 & 8.24 & 7.64 & 415.5 & 0.2750 & 0.20 & 0.21 & 1 \\
\hline NB 1301 & 15.8 .2016 & 26.8 & 7.88 & 3.66 & 350.1 & 0.2223 & 0.16 & 0.14 & 1 \\
\hline NB 1312 & 19.8.2016 & 17.1 & 7.52 & 6.40 & 470.0 & 0.3601 & 0.27 & 0.48 & 2 \\
\hline NB 1318 & 20.8 .2016 & 23.0 & 8.13 & 5.96 & 253.7 & 0.1716 & 0.12 & 0.27 & 3 \\
\hline NB 1330 & 21.8 .2016 & 23.2 & 7.77 & 3.52 & 2385 & 1.5065 & 1.27 & 0.11 & 3 \\
\hline NB 1335 & 21.8 .2016 & 22.7 & 8.90 & 3.35 & 411.0 & 0.2802 & 0.21 & 0.11 & 2 \\
\hline NB 1337 & 21.8 .2016 & 24.5 & 9.01 & 8.89 & 493.1 & 0.3237 & 0.24 & 0.22 & 2 \\
\hline NB 1339 & 22.8 .2016 & 21.2 & 7.82 & 6.78 & 1595 & 1.1180 & 0.87 & 0.66 & 1 \\
\hline NB 1341 & 05.9.2016 & 17.4 & 7.60 & 6.37 & 974 & 0.7410 & 0.57 & 0.46 & 2 \\
\hline NB 1345 & 05.9.2016 & 22.2 & 8.33 & 4.38 & 547 & 0.3770 & 0.28 & 0.21 & 2 \\
\hline NB 1363 & 08.9.2016 & 21.9 & 8.03 & 6.55 & 432.3 & 0.2963 & 0.22 & 0.84 & 3 \\
\hline NB 1367 & 08.9.2016 & 22.1 & 7.97 & 4.83 & 304.6 & 0.2100 & 0.15 & 0.04 & 2 \\
\hline NB 1415 & 11.8 .2017 & 22.0 & 8.65 & 7.91 & 133.3 & 0.0917 & 0.07 & 0.18 & 2 \\
\hline
\end{tabular}

\section{Discussion}

There are two clasping-leaved Potamogeton species growing in Turkey (Uotila 1984, Bayındır 2018). Until recently, $P$. praelongus was thought to be extinct in the country. However, we collected the species from a new location different from that of the historical site in 2016 (Bayındır 2018). Our field observations in the historical site showed that the $P$. perfoliatus is the only species growing here. We identified all recent collections from this historical locality cited in the Flora of Caucasus (Grossheim 1928) as P. perfoliatus. It can be considered that the specimens were incorrectly identified as $P$. praelongus in the past. The other possibility is the replacement of $P$. praelongus from the lake by $P$. perfoliatus. Our recent collection site for $P$. praelongus is from southern Turkey where $P$. perfoliatus does not co-occur (Fig. 1). As indicated previously, $P$. praelongus has a widespread distribution range throughout temperate Northern Hemisphere. However, it is a very rare species in several of the countries it occurs and listed as endangered, critically endangered or near threatened. In several countries, its populations are declining. In Turkey, the species grows in a Mediterranean mountain habitat in an oligotrophic alpine lake at c. $2600 \mathrm{~m}$ (Bayındır 2018). This is the single population for Turkey and the only record for the species in the Mediterranean basin. Distribution range of the population is less than $500 \mathrm{~m}^{2}$. Around the lake where $P$. praelongus grows, there are camping areas for mountain climbers and other tourists. The nearest settlement is $300 \mathrm{~m}$ below the lake, which is used as a summer pasture by villagers for stock farming. Increasing visitors will lead to increased eutrophication of the lake. The species is known to be very sensitive to 
eutrophication and was recorded to disappear in some lakes due to increased eutrophication. Therefore, its unique population is subjected to rapid deterioration due to eutrophication and future climate changes. Potamogeton praelongus is a possible candidate to be negatively affected by global warming due to increase in surface water temperatures since its population is already at $2600 \mathrm{~m}$ (İkinci \& Bayındır 2020). Urgent conservation strategies should be developed for the species to protect its current habitat. In terms of water chemistry preferences, in a study performed throughout Japan, Kadono (1982) found that $P$. praelongus has a $\mathrm{pH}$ range between 7.1 and 7.8 and EC range between 77 and $112\left(\mu \mathrm{S} \mathrm{cm}^{-1}\right)$. We recorded slightly higher EC levels but our $\mathrm{pH}$ measurements fall within the range of this study. Potamogeton praelongus grows in waters with low EC, low salinity and low ammonium concentrations (Table 2 ). These are expected results since the alpine lake is mainly fed by snowmelt and rainwater. Other macrophytes occurring in the same lake are P. natans, Stuckenia filiformis and Persicaria amphibia. Among these species, S. filiformis is also very rare in Turkey and has only one additional record from Eğrigöl Lake in Antalya at $2060 \mathrm{~m}$ altitude. Different studies classify Eğrigöl Lake as either oligotrophic or oligo-mesotrophic (Kaymakçı-Başaran \& Egemen 2006, Aygen et al. 2009). On the other hand, $P$. natans is a widespread species throughout Turkey with a tendency of preferring higher altitudes (İkinci \& Bayındır 2020). Persicaria amphibia is a very widespread species having natural distribution range as northern circumpolar temperate but it is naturalized in North and South America, and South Africa (Partridge 2001). It is also a very common species in Turkey (Seçmen \& Leblebici 2008, İkinci \& Bayındır 2019). It can be found in different habitats including disturbed ones. It is not found in fast flowing water bodies. The species has adaptation to water level fluctuations because it has both aquatic and terrestrial forms (Partridge 2001). Persicaria amphibia occurs in a wide range of altitude and it was also found at higher altitudes in the Himalayas similar to Turkish population mentioned in our current study (Ram et al. 1989).

Potamogeton perfoliatus has almost a cosmopolitan distribution in the world (Gupta \& Lansdown 2013). In Turkey, we found records of the species from 54 wetlands based on examination of 86 herbarium specimens (Fig. 1). In addition to the recent Illustrated Flora of Turkey (Bayındir 2018), we added 5 new wetlands for the distribution of the species based on our collections (Table 1). Therefore, we can better understand the extent of occurrence of $P$. perfoliatus in Turkey. The species has a broad altitudinal distribution from sea level to $2450 \mathrm{~m}$ but majority of collections are below $1200 \mathrm{~m}$ (İkinci \& Bayındır 2020). Water chemistry measurements were performed from 24 sampling sites for the species (Table 4). It grows in water bodies with high $\mathrm{pH}$ levels. Majority of the collection sites are inland freshwaters. However, we recorded the highest salinity from an inland lake (Hafik Lake) in Sivas. We found the species in lakes and canals close to the Black Sea and the Aegean Sea but still with low salinity levels (max. $1.10 \mathrm{ppt}$ ). These findings are parallel to previous reports about $P$. perfoliatus (Burns et al. 1995). According to Twilley et al. (1985), $P$. perfoliatus grows in oligohaline to mesohaline waters. We recorded $P$. perfoliatus mainly from lentic habitats, out of 54 wetlands only seven were fast flowing rivers and canals. Our collection sites for the species were not shallow waters because the submersed broad-leaved species generally cannot tolerate drawdowns. When we analyzed the other accompanying Potamogetonaceae species provided in Table 1, we see the highest cooccurrence with Stuckenia pectinata in 18 wetlands. Stuckenia pectinata is the most widespread Potamogetonaceae species in Turkey which we sampled from 65 wetlands (İkinci \& Bayındır 2020). Other species with the highest number of co-occurrences with $P$. perfoliatus are $P$. crispus, $P$. nodosus, and $P$. berchtoldii, respectively. These are also common species which we collected from more than 30 wetlands in Turkey (İkinci \& Bayındır 2020). On the other hand, $P$. natans was sampled only in two common wetlands with $P$. perfoliatus (Table 1 ). We collected $P$. natans from 11 wetlands in Turkey which are mainly above $1200 \mathrm{~m}$, indicating a clear difference in altitudinal preference compared to $P$. perfoliatus (İkinci \& Bayındır 2018). The other interesting result is that $P$. perfoliatus were not found in water bodies where seven rare Potamogetonaceae species are growing (Bayındır 2018, İkinci \& Bayındır 2020).

There are clear morphological and anatomical differences between $P$. praelongus and $P$. perfoliatus. Zig-zag shaped stem, cucullate leaf apice, leaf base, leaf margins and conspicuous, long, persistent stipule, presence of interlacunar bundles, subepidermal bundles and shape of endodermal cells in stem discriminate two clasping-leaved Potamogeton species (Table 3 and 5). Persistence of the stipule is an important character in the identification of Potamogeton species. In the newly collected $P$. praelongus specimens, stipules are persistent until the late stages of plants life cycle. However, in $P$. perfoliatus specimens we collected stipules decay early during the developmental stages of the plants and therefore are not persistent (Table 5). Previous studies indicated that leaf length is clearly longer in P. praelongus (Hagström 1916, Ogden 1943, Haynes 1985, Wiegleb \& Kaplan 1998). In our samples, we measured maximum leaf length as $170 \mathrm{~mm}$ for $P$. praelongus and as $61 \mathrm{~mm}$ for $P$. perfoliatus (Table 5). 
Table 5. Comparison of morphological characters of $P$. praelongus and $P$. perfoliatus based on our collections and former reports on the two species.

\begin{tabular}{|c|c|c|c|c|c|c|}
\hline & & \multicolumn{5}{|c|}{ Character } \\
\hline & & Stem & Leaf base & Leaf apice & Stipule & $\begin{array}{l}\text { Maximum leaf } \\
\text { lenght }(\mathrm{mm})\end{array}$ \\
\hline \multirow{2}{*}{ This study } & P. praelongus & Zig-zag & Semiamplexicaule & Cucullate & $\begin{array}{l}\text { Conspicuous, } \\
\text { long, persistent }\end{array}$ & 170 \\
\hline & P. perfoliatus & Straight & Amplexicaul & $\begin{array}{c}\text { Rarely } \\
\text { cucullate }\end{array}$ & Nonpersistent & 61 \\
\hline \multirow{2}{*}{$\begin{array}{l}\text { Hagström } \\
\text { (1916) }\end{array}$} & P. praelongus & - & Hooded & $\begin{array}{l}\text { Always } \\
\text { cucullate }\end{array}$ & Persistent & $200-250$ \\
\hline & $P$. perfoliatus & - & $\begin{array}{c}\text { Cordate- } \\
\text { amplexicaul }\end{array}$ & $\begin{array}{l}\text { Slightly } \\
\text { recurved }\end{array}$ & Fugacious & - \\
\hline \multirow{2}{*}{ Ogden (1943) } & P. praelongus & Often zigzag & $\begin{array}{c}\text { Clasping about } \\
1 / 4 \text { or } 2 / 4\end{array}$ & Cucullate & Persistent & $200(-360)$ \\
\hline & $P$. perfoliatus & - & $\begin{array}{l}\text { Clasping about } \\
3 / 4\end{array}$ & Noncucullate & $\begin{array}{l}\text { Delicate, } \\
\text { fugacious }\end{array}$ & $60(-70)$ \\
\hline \multirow[b]{2}{*}{ Haynes (1985) } & P. praelongus & Zig-zag & Semi-clasping & Cucullate & Persistent & $80-280$ \\
\hline & $P$. perfoliatus & - & Clasping & $\begin{array}{l}\text { Non-cucullate } \\
\text { (flattened) }\end{array}$ & $\begin{array}{l}\text { Stipules } \\
\text { delicate }\end{array}$ & $76(-97)$ \\
\hline \multirow{2}{*}{$\begin{array}{c}\text { Kaplan \& } \\
\text { Wiegleb } \\
(1998)\end{array}$} & P. praelongus & - & Semiamplexicaul & $\begin{array}{c}\text { Distinctly } \\
\text { hooded }\end{array}$ & $\begin{array}{l}\text { Long, } \\
\text { persistent }\end{array}$ & $180(-360)$ \\
\hline & P. perfoliatus & - & Amplexicaul & $\begin{array}{l}\text { Slightly } \\
\text { hooded }\end{array}$ & Decaying early & 115 \\
\hline
\end{tabular}

As regarding the anatomical differences of the two species, in majority of the previous studies (Raunkier 1903, Hagström 1916) and in our samples, interlacunar bundles and subepidermal bundles are present in $P$. praelongus specimens (Table 3 ). In $P$. perfoliatus both interlacunar and subepidermal bundles are either not found or rarely occur in specimens with one or a few small bundles (Raunkier 1903, Wiegleb \& Kaplan 1998). Our studies are in parallel to these findings (Figs. 2, 3). Hagström (1916) stated that $P$. praelongus have sclerenchyma cells which we also observed (Fig. 3). In contrast to previous studies (Raunkier 1903, Hagström 1916, Haynes 1985, Wiegleb 1990, Wiegleb \& Kaplan 1998), we found trio type stele, not the proto type in $P$. praelongus. Such differences may occur among the populations of very widespread species. Our results showed that steles of our specimens are made up of four median bundles, three of which are united. We found that pseudohypodermis is uniseriate in both species. As a result, it can be stated that the presence interlacunar bundles and sclerenchyma cells are useful anatomical characters to distinguish these two species.

\section{References}

1. Ascherson, P. \& Graebner, P. 1907. Potamogetonaceae. Das Pflanzenreich, Regni vegetabilis conspectus, 31: 39142.

2. Aygen, C., Mis, D.Ö., Ustaoğlu, R.M. \& Balik, S. 2009. Zooplankton composition and abundance in Lake Eğrigöl, a high mountain lake (Gündoğmuş, Antalya). Turkish Journal of Zoology, 33: 83-88. https://doi:10.3906/zoo0805-11.

3. Aykurt, C., Fehrer, J., Sarı, D., Kaplan, Z., Deniz, İ. G., Aydemir, E. \& İmir, N. 2017. Hybridization between the
In this study, we present information about the physical and chemical properties of the water where $P$. praelongus and $P$. perfoliatus grow and their other habitat requirements. We give a detailed distribution of the two species based on a broad sampling covering entire Turkey. We also provide new morphological descriptions and the first anatomical studies of the species from Turkey. One of these species, $P$. praelongus is critically endangered (CR) in Turkey and was recorded only from a single site. The other species, $P$. perfoliatus is widespread throughout Turkey occurring under different environmental conditions. As we analyzed the habitat preferences of the species, our study provides useful information for future monitoring of the two species in terms of conservation strategies.

\section{Acknowledgement}

This study was funded by Bolu Abant Izzet Baysal University Scientific Research Projects Coordination Unit (Project no: 2015.03.01.895). ANG Foundation provided a three-year grant for the first author to make a revision of the Potamogetonaceae family in Turkey and supported herbarium visits.

linear-leaved Potamogeton species in Turkey. Aquatic Botany, 141: 22-28.

4. Bayındır, N. 2018. "Potamogeton L.,"In Resimli Türkiye Floras1 Cilt 2 (Illustrated Flora of Turkey Volume 2), İstanbul: Ang Vakfi Nezahat Gökyiğit Botanik Bahçesi Yayınlar1, pp. 636-664.

5. Bayındır, N. \& İkinci, N. 2020a. The role of environmental variables on distribution of Potamogetonaceae species. Wetlands, 40(1): 125-133. https://doi:10.1007/s13157-01901163-w. 
6. Bayındır, N. \& İkinci, N. 2020b. Althenia orientalis (Potamogetonaceae) in Turkey: Habitat conditions, morphology and anatomy. Communications Faculty of Sciences University of Ankara Series C Biology, 29(2): 243-253.

7. Bornette, G. \& Puijalon, S. 2011. Response of aquatic plants to abiotic factors: a review. Aquatic Sciences, 73: 114.

8. Burns, J.W., Michael J.R., Poirrier, A. \& Preston, K.P. 1995. The status of Potamogeton perfoliatus (Potamogetonaceae) in Lake Pontchartrain, Louisiana. Sida, 16: 757-763.

9. Crisp, D.J. \& Southward, A.J. 1958. The distribution of intertidal organisms along the coasts of the English Channel. Journal of the Marine Biological Association of the United Kingdom, 37(1): 157-203.

10. Dunham, A., Archer, S.K., Davies, S.C., Burke, L.A., Mossman, J., Pegg, J.R. \& Archer, E. 2018. Assessing condition and ecological role of deep-water biogenic habitats: Glass sponge reefs in the Salish Sea. Marine environmental research, 141: 88-99.

11. Ergüner, Y., Kumar, J., Hoffman, F.M., Dalfes, H.N. \& Hargrove, W.W. 2019. Mapping ecoregions under climate change: a case study from the biological 'crossroads' of three continents, Turkey. Landscape Ecology, 34: 35-50. https://doi.org/10.1007/s10980-018-0743-8.

12. Grossheim, A.A. 1928. Flora Kavkaza. Vol 1. Narodnyi Komissariat Zemledeliya SSR Armenii, Tiflis, 295 pp.

13. Guo, J.L., Yu, Y.H., Zhang, J.W., Li, Z.M., Zhang, Y.H. \& Volis, S. 2019. Conservation strategy for aquatic plants: endangered Ottelia acuminata (Hydrocharitaceae) as a case study. Biodiversity and Conservation, 28: 1533-1548. https://doi.org/10.1007/s10531-019-01740-9.

14. Gupta, A.K. \& Lansdown, R.V. 2013. Potamogeton perfoliatus. The IUCN Red List of Threatened Species: e.T164189A1029195. http://dx.doi.org/10.2305/IUCN.UK.2013-

1.RLTS.T164189A1029195.en. (Date accessed: 09.12.2019)

15. Hagström, J.O. 1916. Critical researches on the Potamogetons. Kungl. Svenska Vetenskapsakad Handl, 55(5): 1-281.

16. Haynes, R.R. 1985. A revision of the clasping-leaved Potamogeton (Potamogetonaceae). Sida, 11: 173-188.

17. İkinci, N. \& Bayındır, N. 2019. Distribution and Richness of Aquatic Plants of Bolu Province (Turkey). Fresenius Environmental Bulletin, 28: 244-256.

18. İkinci, N. \& Bayındır, N. 2020. Spatial trends of Potamogetonaceae along an altitudinal gradient. Biologia, https://doi.org: 10.2478/s11756-020-00596-7.

19. IUCN. 2012. IUCN Red List of Threatened Species. Version 2011.2 http://www.iucnredlist.org/ (Date accessed 16.12.2019).

20. Julve, P.H. 2017. Potamogeton praelongus Wulfen. Baseflor. Index botanique, écologique et chorologique de la flore de France. http//www.tela-botanica.org. (Date accessed: 09.02.2017).
21. Kadono, Y. 1982. Distribution and habitat of Japanese Potamogeton. Botanical Magazin Tokyo, 95: 63-76.

22. Kaplan, Z., Jarolímová, V. \& Fehrer, J. 2013. Revision of chromosome numbers of Potamogetonaceae: A new basis for taxonomic and evolutionary implications. Preslia, 85: 421-482.

23. Kaymakçı-Başaran, A. \& Egemen, Ö. 2006. Orta Toros Dağlarındaki Eğrigöl'ün su kalitesi parametrelerinin araştırılması. Tarım Bilimleri Dergisi, 12: 137-143.

24. Lansdown, R.V. 2014. Potamogeton praelongus. The IUCN Red List of Threatened Species 2014: e.T167906A42402297. http://dx.doi.org/10.2305/IUCN.UK.20141.RLTS.T167906A42402297.en (Date accessed: 21.01.2018).

25. Ogden, E.C. 1943. The broadleaved species of Potamogeton of North America north of Mexico. Rhodora, 45: 57-105, 119-163, 171-214.

26. Partridge, J.W. 2001. Persicaria amphibia (L.) Gray (Polygonum amphibium L.). Journal of Ecology, 89(3): 487-501.

27. Prausová, R., Janová, J. \& Adamac, L. 2011. Rescue of the critically endangered long-stalked pondweed (Potamogeton praelongus) in the Czech Republic. Acta Biologica Slovenica, 54: 43-54.

28. Prausová, R., Sikorová, P. \& Šafářová, L. 2014. Generative reproduction of long stalked pondweed (Potamogeton praelongus Wulfen) in the laboratory. Aquatic Botany, 120: 268-274.

29. Ram, J., Singh, J.S. \& Singh, S.P. 1989. Plant biomass, species diversity and net primary production in a central Himalayan high altitude grassland. The Journal of Ecology, 77(2): 456-468.

30. Raunkier, C. 1903. Anatomical Potamogeton studies and Potamogeton fluitans. Botanisk Tidsskrift, 25: 253-280.

31. Seçmen, Ö. \& Leblebici, E. Ö. 2008. Türkiye Sulak Alan Bitkileri ve Bitki Örtüsü (2. Bask1), İzmir: Ege Üniversitesi Yayınları, pp. 57.

32. Thiers, B. 2016. Index Herbariorum: A global directory of public herbaria and associated staff. New York Botanical Garden's Virtual Herbarium. Website http://sweetgum.nybg.org/science/ih (Date accessed: 04.03.2020)

33. Twilley, R.R., Kemp, W.M., Staver, K.W., Stevenson, J C. \& Boynton, W.R. 1985. Nutrient enrichment of estuarine submersed vascular plant communities. 1. Algal growth and effects on production of plants and associated communities. Marine ecological progress series. Oldendorf, 23: 179-191.

34. Uotila, P. 1984. Potamogeton L., 17-28. In: Davis PH, Mill RR Mill, Tan K, (eds). Flora of Turkey and the East Aegean Islands. Vol. 8. Edinburgh University Press, Edinburgh, $632 \mathrm{pp}$.

35. Vöge, M. 1992. Die Entwicklung von Potamogeton praelongus im Großensee bei Hamburg. Tuexenia, 12: 275 284. 
36. Wiegleb, G. 1990. The importance of stem anatomical characters for the systematics of the genus Potamogeton L. Flora, 184: 197-208.

37. Wiegleb, G. \& Kaplan Z. 1998. An account of the species of Potamogeton L. (Potamogetonaceae). Folia Geobotica, 33: $241-316$
38. Wiegleb, G. 1988. Notes on pondweeds- outlines for a monographical treatment of the genus Potamogeton L. Feddes Repertorium, 99: 249-266. 